http://jmscr.igmpublication.org/home/ ISSN (e)-2347-176x ISSN (p) 2455-0450

crossref DOI: https://dx.doi.org/10.18535/jmscr/v9i10.02

\title{
Role of Multi-detector Row (320) CT Coronary Angiography in Assessment of Coronary Stents in Comparison with Coronary Angiography
}

\author{
Authors \\ Amr Asem Ali Omran ${ }^{*}$, Ashraf Mohammed Enite ${ }^{2}$, Emam Mohammed Abd-Elaziz \\ ${ }^{1}$ Senior Radiologist, Department of Radiodiagnosis, Police Authority Hospital-Cairo \\ ${ }^{2}$ Professor, Department of Radiodiagnosis, Faculty of Medicine - Al-Azhar University-Cairo \\ ${ }^{3}$ Professor and HOD, Department of Radiodiagnosis, Faculty of Medicine - Al-Azhar University- Cairo \\ *Corresponding Author
}

Amr Asem Ali Omran

Senior Radiologist, Department of Radiodiagnosis, Police Authority Hospital-Cairo

\begin{abstract}
Background: Coronary artery disease $(C A D)$ is the leading cause of morbidity and mortality in industrialized society. To treat patients with obstructive atherosclerosis, percutaneous coronary intervention with stent implantation is routinely performed, which considerably reduces the rate of restenosis as compared with balloon angioplasty.

Aim of the Work: to evaluate multi-detector CT angiography as a less invasive technique in the assessment of the coronary stent patency and compared with the conventional angiography findings (as a gold standard technique).

Patients and Methods: This study included 50 patients with prior coronary stent deployment, 32 of them (64\%) underwent conventional angiography as a gold standard for evaluation of the patency of the coronary stents. The indications of conventional angiography were unstable angina in 10 cases out of the 32 cases (31.25\%), while the rest were performed to assess stent patency after suspected instent re-stenosis or atypical chest pain in 22 cases out of $32(68.75 \%)$.

Results: In this study CT angiography compared to the conventional angiography as a gold standard technique gave us a sensitivity of $92.3 \%$, a specificity of about $100 \%$, an accuracy of about $95.6 \%$, PPV of $100 \%$ and NPV of $90.5 \%$ as regarding patent stent taking into consideration that 2 stents were non evaluable due to narrow stent caliber $(2.5 \mathrm{~mm}$.) but proved patent by conventional angiography, but these results will much improved if the non evaluable stents removed from statistical analysis.

Conclusion: Our study recommends usage of latest multi-detector row CT scanners as a first-line tool for the noninvasive evaluation of patients with suspected instent restenosis especially with stents diameter $\geq$ $3 \mathrm{~mm}$. and helps to identify factors that influence the assess ability of coronary artery stents by $320 \mathrm{MDCT}$ scanners, namely, stent type and diameter.
\end{abstract}

Keywords: Multi-detector Row (320) - CT Coronary Angiography - Coronary Stents.

\section{Introduction}

Coronary artery disease (CAD) is the leading cause of morbidity and mortality in industrialized society. To treat patients with obstructive atherosclerosis, percutaneous coronary intervention with stent implantation is routinely performed, which considerably reduces the rate of restenosis as compared with balloon angioplasty. Nevertheless, also 
in patients treated with coronary stent implantation, a risk of in-stent restenosis remains. Specifically, stent implantation may be followed by early in-stent thrombosis or later occurring in-stent restenosis due to neointimal hyperplasia. Early detection and treatment of in-stent restenosis is of vital importance, as it may help reduce morbidity and mortality. Considering an increasingly growing number of patients with coronary stent implantation, in combination with the potential risk of in-stent restenosis, a noninvasive approach for the detection of in-stent restenosis would be desirable ${ }^{(1)}$.

With the introduction of multidetector computed tomography coronary angiography (CTA), the noninvasive assessment of the coronary artery tree has become feasible. Although early 4-row CTA systems did not yet enable the evaluation of stented coronary segments with improved spatial and temporal resolutions of 16- row and 64-row systems the assessment of in-stent restenosis became feasible. Despite increased temporal and spatial resolution of 64-row CTA, a proportion of stents remained uninterpretable, predominantly because of cardiac motion artifacts as well as high density artifacts caused by the metallic stent struts ${ }^{(1)}$.

A new generation of CTA scanners became available with 320 simultaneous detector-rows each $0.5 \mathrm{~mm}$ wide and increased temporal resolution as compared with most of the previous scanner generations. Because of superior craniocaudal coverage up to $16 \mathrm{~cm}$, these systems allow a volumetric scanning approach, covering the entire heart in a single gantry rotation or heart beat. Single heart beat image acquisition has some advantages over scanning techniques requiring multiple heart beats. First, the reduced scan time shortens the time of breath-hold and lowers the amount of contrast material. Moreover, volumetric scanning decreases patient radiation exposure by eliminating helical oversampling and furthermore eliminates the problem of stair-step artifacts, observed in helical or step-and-shoot scanning techniques. Although the diagnostic accuracy of 320-row CTA in the anatomic assessment of significant CAD has been recently reported ${ }^{(1)}$.

\section{Aim of the Work}

The aim of this study is to evaluate multi-detector CT angiography as a less invasive technique in the assessment of the coronary stent patency and compared with the conventional angiography findings (as a gold standard technique).

\section{Patients and Methods}

\section{Patients}

Study Population: A total number of 50 patients were enrolled for multi-detector row CT angiography of the coronary arteries between October 2015 and May 2018. 32 patients underwent both conventional coronary angiography and MDCT coronary angiography. Coranary angiography was done within a month after MDCT examination. The patients who did not perform the conventional angiography were mostly due to the decision made by the referring physicians as there is increased confidence in the results of MSCT angiography, so those patients were referred for conservative medical treatment. Written informed consent was obtained from all patients participated in this study after full explanation of the benefits and risks of the procedure. They were informed about any unexpected risks that may appear during the course of this study. All patients' related information were kept confidential. Fourty patients (80\%) presented with recurrent chest pain (defined as a retro-sternal or precordial diffuse burning, heaviness, or squeezing sensation that may radiate to the left arm, neck or lower jaw and is precipitated by effort and relived by rest or nitrates) or suspected progression of known coronary artery disease. While the remaining 10 patient's (20\%) came for regular follow up to assess the patency of previously deployed coronary stents by MSCT. The patients mean age was 56 years old $\pm \mathrm{SD}$, ranging between 39 and 73 years old. There were a total number of 43 males and 7 females. The average heart rate was 62 beats/min.

The inclusion criteria included: Patients having recurrent chest pain with previous history of coronary stent insertion. Need to assess the progression of the disease in the native arteries. 
Asymptomatic patient underwent previous coronary stenting and shows positive results for myocardial ischemia during regular follow-up on doing noninvasive tests such as thallium scan, stress echocardiography or stress ECG.

The exclusion criteria were: Arrhythmias. Heart rate above $80 / \mathrm{min}$ not responding to medical preparation. Clinically unfit patients (unable to stop breathing during the examination).

\section{METHODS}

Instructions: Patients were asked to fast 4-6 hours prior to the examination. Medications are not to be discontinued. Avoid caffeine products and smoking and exercise one day before scan. Stop taking phosphodiesterase inhibitors used to treat erectile dysfunction or pulmonary hypertension.

Patient Preparation: The heart rate was evaluated before the examination. The examination was done if the heart rate is below 65 beats per minute. Patients with heart rates above 70 beats per minute were given cardio-selective beta-blocker; $100 \mathrm{mg}$ of Metoprolol or atenolol orally 1 hour before the study to obtain a stable low heart rate, provided that contra-indications to $\beta$-blockers are excluded.

At scanner room: Explanation of procedure with reassurance to relief anxiety. Patients dressed a gown and then instructed to lie supine on scanner table with arms raised above their heads. ECG electrodes were applied to chest wall after skin preparation with alcohol and ECG trace was monitored. Intra-venous canulla (16-22 g.) in right anticubital vein was connected and test injection with saline was done. $5.0 \mathrm{mg}$ sublingual Isosorbid dinitrate was given. The steps of the study were explained in details for each patient. To evaluate patients ability of breath-withholding; they were required to perform a deep inspiration and to continue to hold their breath without pushing (i.e. Valsalva maneuver). During this trial, the patient was observed for compliance and the electrocardiogram for significant changes.

Contrast Material: A bolus of $70-80 \mathrm{ml}$ of water soluble non-ionic contrast (Ultravist $370 \mathrm{mg}$ Schering, Berlin, Germany) was injected through canula into an upper limb vein (right antecubital vein in all cases to reduce left sided artifacts) with a flow rate of 5 to 5.5 $\mathrm{mL} / \mathrm{sec}$. This injection was automatically followed by injection of about 40-50 $\mathrm{cc}$ of saline at a flow rate of 4 $\mathrm{ml} / \mathrm{sec}$ using a programmed dual head power injector pump (MedRad; USA) to maintain good opacification of the coronary vessels with wash out of contrast material from the SVC and right side of the heart that may cause artifacts.

Scan Protocol: All patients were scanned with 320-row multidetector CT scanner (Aquilion One, Toshiba Medical Systems, Otawara, Japan) installed at police authority Hospitals through these steps:

Initial scanogram AP and lateral projection for automatic radiation dose calculation and for planning scan range from carina down to the apex of the heart.

Automatic bolus tracking technique was used to detect the arrival of contrast material at descending aorta. The ROI was placed at descending aorta at mid heart level with trigger threshold set at $180 \mathrm{HU}$. Repetitive low-dose monitoring examinations (120 $\mathrm{kV}, 50 \mathrm{mAs}, 0.5$-s scanning time) were performed $10 \mathrm{~s}$ after contrast medium injection began. When trigger threshold was reached, scan started immediately after breath holding command.

Image acquisition: Acquisition parameters: $0.35 \mathrm{~s}$ gantry rotation time, variable $\mathrm{mA}$ according to patient body habitus (range: $250-580 \mathrm{~mA}$ ), variable $\mathrm{kv}$ according to patient body habitus (range: $80-135$ $\mathrm{kv})$.

Prospective ECG gating was used with volume scanning method. Single heart beat acquisition was routinely performed in those with heart rate below $65 \mathrm{bpm}$ and the scan window was set at $70-80 \%$ of $\mathrm{R}-\mathrm{R}$ interval. In those with heart rate ranged from 65 to $70 \mathrm{bpm}$, the scanning window was set to 30 $80 \%$ of R-R interval to include end systolic phase.

Heart rate remained above $70 \mathrm{bpm}$ at four $(16.7 \%)$ patients due to suboptimal response to oral medications used and anxiety. In those patients, CT acquisition was done using multiple heart beats to improve temporal resolution with scanning window set manually to cover $30-80 \%$ of the R-R interval. 
Image-reconstruction: Images were reconstructed at $0.5 \mathrm{~mm}$ slice thickness and $0.5 \mathrm{~mm}$ interval with smooth and sharp reconstruction kernels (FC03 \& FC05 respectively) at $75 \%$ of $\mathrm{R}-\mathrm{R}$ interval and at the best diastolic phase. The scan field of view was set to as small as possible for better spatial resolution. Post processing: The reconstructed images were transferred to workstation (Vitrea Fx, Vital Images, Japan) to form multiplanar reformatted images in axial, sagittal and coronal planes. Also maximum intensity projection, 3D volume rendered images and curved planar reformations were obtained.

\section{Image-analysis}

Assessment of image quality: A stent was considered assessable when the stent lumen was visible and contrast attenuation of the lumen could be evaluated qualitatively without the influence of partial volume effects, metal artifacts of stents, or cardiac motion artifacts. Each stent was assigned an image quality score of $\mathbf{1}$ (good image quality, no artifacts affecting evaluation of the stent), $\mathbf{2}$ (adequate image quality, mild to moderate artifacts, blurring but acceptable for clinical diagnosis), or $\mathbf{3}$ (poor image quality, uninterpretable with severe artifacts making stent evaluation impossible) according to the criteria used for assessability. Reduced image quality was evaluated in relation to stent location, diameter and strut thickness.

Assessment of stent lumen: Stent lumen was assessed at workstation using curved planar reformatted images while $3 \mathrm{D}$ volume rendered images were used for global assessment of stent location and native coronary arteries.

\section{Each stent was defined as follows:}

Patent: with no visible neointimal hyperplasia: the absence of low attenuation areas between stent wall and contrast enhanced lumen.

\section{Patent with insignificant neointimal hyperplasia:}

longitudinal low-attenuation areas along the stent wall observed as a rim of hypo attenuation between the stent and the contrast enhanced vessel lumen exerting $<50 \%$ stenosis.
Patent with in-stent restenosis: longitudinal and transverse low-attenuation areas along the stent wall exerting $\geq 50 \%$ stenosis.

In-stent occlusion: the complete absence of contrast material within stent lumen.

Evaluation of non-stented coronary arteries: The non-stented coronary arteries were also evaluated to detect areas of significant stenosis $(\geq 50)$ or occlusion that may contribute to recurrent patient's symptoms. The lumen assessment was done on looking through $3.0 \mathrm{~mm}$ axial MIP images and curved planar reformatted images.

Conventional coronary angiography was done in 32 cases after MDCT on request of cardiologist and cardio-thoracic surgeon for confirmation and comparison.

Statistical Analysis SPSS version 21 (IBM Inc.) was used which included the following: $T$ test for comparison of means for numerical scaling variables e.g. body measurements. Cross tabulation with Fisher's exact test of significance for comparison of the nominal variables. F statistics for test of variance between and within our studied groups. In all these tests, the statistical significance was considered at $5 \%$ level if $(\mathrm{P}<0.05)$.

\section{Results}

The mean age of the included patients was 56 with an age range between 39 and 73 years. Male patients were $43(86 \%)$ while females were 7 (14\%), Table (1).

Table (1): Cases demographic data.

\begin{tabular}{|l|c|c|}
\hline \multicolumn{2}{|c|}{} & No. of cases =50 \\
\hline \multirow{2}{*}{ Age } & Mean \pm SD & $56.22 \pm 9.21$ \\
& Range & $39-73$ \\
\hline \multirow{2}{*}{ Sex } & Females & $7(14.0 \%)$ \\
& Males & $43(86.0 \%)$ \\
\hline \multirow{2}{*}{ Weight } & Mean \pm SD & $87.40 \pm 11.93$ \\
& Range & $61-120$ \\
\hline \multirow{2}{*}{ Length } & Mean \pm SD & $171.16 \pm 5.80$ \\
& Range & $155-179$ \\
\hline \multirow{2}{*}{ BMI } & Mean \pm SD & $30.25 \pm 4.21$ \\
& Range & $19.6-39.63$ \\
\hline \multirow{2}{*}{ Systolic BP } & Mean \pm SD & $133.20 \pm 12.32$ \\
& Range & $100-160$ \\
\hline \multirow{2}{*}{ Diastolic BP } & Mean \pm SD & $82.70 \pm 10.11$ \\
& Range & $60-100$ \\
\hline
\end{tabular}

A total of 65 coronary artery stents deployed within 50 patients were included in this study; and assessed by 
MDCT for follow up of their patency, 45 stents in 32 cases (56.25\% of the total included stents) underwent conventional coronary angiography and after correlation of the MDCT with the conventional coronary angiography, they were classified as follows:

- 2 stents were non-evaluable by MDCT

○ Both of them is $(2.5 \mathrm{~mm})$ in caliber due to heavy stent struts and narrow caliber and proved to be patent stent by conventional angiography.

- Within the 43 evaluable stents the following results were found, Table (2):

1) 8 stents: were reported to be totally occluded by 320 MDCT and proved their occlusion by conventional coronary angiography [their calibers are: three stents of $(3 \mathrm{~mm})$, and five stents of (3.5mm.)

2) 11 stents: were reported to have suspected instent restenosis by MDCT as following:

A- Nine stents $\geq 50 \%$ stenosis and the conventional coronary angiography revealed that 7 of them have instent re-stenosis $\geq 50 \%$ [ their calibers are: two stents of (3mm.), four stents of $(3.5 \mathrm{~mm})$ and one of $(4 \mathrm{~mm})]$, while 2 stents proved to be $30 \%$ stenosed by angiography both of (3 $\mathrm{mm}$.)

B- Two stents $<50 \%$ stenosis and the conventional coronary angiography revealed that both of them are $30 \%$ stenosis [their calibers are: one stent of $(3 \mathrm{~mm}$.) and one stent of $(3.5 \mathrm{~mm})]$

3) 24 stents: were reported to be patent by MDCT and proved their patency by conventional coronary angiography after re-checking due to clinical conditions recommending so in the form recurrent chest pain and unstable angina [ their calibers are: eight of $(3 \mathrm{~mm})$, ten of $(3.5 \mathrm{~mm})$,

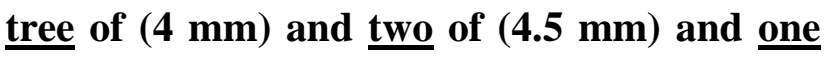
of (5 mm.)],
Table (2): Relation between the results of MSCT and conventional coronary angiography in the evaluated 45 stents in 32 patients

\begin{tabular}{|l|c|c|c|c|c|}
\hline \multirow{2}{*}{$\begin{array}{l}\text { Status of } \\
\text { 45 stents }\end{array}$} & $\begin{array}{c}\text { Assessment of } \\
\text { 45 stents } \\
\text { by CT }\end{array}$ & $\begin{array}{c}\text { Assessment 45 } \\
\text { stents } \\
\text { by angio }\end{array}$ & \multicolumn{3}{|c|}{ Chi-square test } \\
\cline { 2 - 6 } & No. (\%) & No. (\%) & $\mathbf{X}^{2}$ & $\begin{array}{c}\text { P- } \\
\text { value }\end{array}$ & Sig. \\
\hline Patent & $24(53.3 \%)$ & $26(57.8 \%)$ & 0.180 & 0.671 & NS \\
\hline Occluded & $8(17.8 \%)$ & $8(17.8 \%)$ & 0.000 & 1.000 & NS \\
\hline $\begin{array}{l}\geq \quad 50 \% \\
\text { stenosis }\end{array}$ & $9(20.0 \%)$ & $7(15.6 \%)$ & 0.304 & 0.581 & NS \\
\hline $\begin{array}{l}<\quad 50 \% \\
\text { stenosis }\end{array}$ & $2(4.4 \%)$ & $4(8.9 \%)$ & 0.714 & 0.398 & NS \\
\hline
\end{tabular}

P-value > 0.05: Non significant; P-value < 0.05: Significant; Pvalue $<0.01$ : Highly significant

The calibers of the deployed coronary stents in our study ranging from 2.5 up to $5 \mathrm{~mm}$ and so if we classify our results of the evaluated 45 stents by both MDCT and conventional angiography in correlation to the caliber of the deployed stents, the results will be as follows:

- 2 stents of $2.5 \mathrm{~mm}$ in caliber: Both were nonevaluable by MDCT due to narrow stent caliber and heavy struts proved patent by angiography.

- 16 stents of $3 \mathrm{~mm}$ in caliber: Eight stents were patent by $\mathrm{CT}$ and proved patent by angiography, three stents were occluded by both CT and catheter angiography, two stents were stenosed $\geq 50 \%$ by CT and proved stenosed $50 \%$ by angiography, two stents were stenosed $\geq 50 \%$ by CT and while proved stenosed $30 \%$ by angiography, one stent was stenosed $<50 \%$ by CT and proved stenosed $30 \%$ by angiography.

- 20 stents of $3.5 \mathrm{~mm}$ in caliber: 10 stents were patent by both CT and catheter angiography, $\mathbf{5}$ stents were occluded by both $\mathrm{CT}$ and angiography, 4 stents were suspected to be restenosed $\geq 50 \%$ by $\mathrm{CT}$ and proved also $>50 \%$ stenosed by conventional angiography and one stent was suspected to be re-stenosed $<50 \%$ by CT and proved also $30 \%$ stenosed by conventional angiography.

- 4 stents of $4 \mathrm{~mm}$ in caliber: 3 stents were patent by both CT and catheter angiography, 1 stent was restenosed $\geq 50 \%$ by CT and proved $>50 \%$ stenosis by conventional angiography. 
- 2 stents of $\mathbf{4 . 5} \mathrm{mm}$ in caliber: Both were patent by both CT and catheter angiography.

- 1 stent of $5 \mathrm{~mm}$ in caliber: was patent by both $\mathrm{CT}$ and catheter angiography.

Table (3): Difference between 2 tests and reation to stent diameter

\begin{tabular}{|l|c|c|c|c|c|}
\hline \multirow{2}{*}{$\begin{array}{l}\text { Diameter of } \\
\text { stent } \\
(\mathbf{m m})\end{array}$} & \multicolumn{2}{|c|}{$\begin{array}{c}\text { Difference between 2 } \\
\text { tests }\end{array}$} & \multirow{3}{*}{$\begin{array}{c}\text { Test } \\
\text { value• }\end{array}$} & $\begin{array}{c}\text { P- } \\
\text { value }\end{array}$ & \multirow{2}{*}{ Sig. } \\
\cline { 2 - 3 } & No & Yes & & \\
\cline { 2 - 3 } & No. $=\mathbf{4 1}$ & No. $=\mathbf{4}$ & & \\
Mean \pm SD & $3.46 \pm 0.47$ & $\begin{array}{c}2.75 \pm \\
0.29\end{array}$ & 2.986 & 0.005 & HS \\
\hline Range & $3-5$ & $2.5-3$ & & & \\
\hline
\end{tabular}

Table (3) demonstrates when the callipar of stent $<$ $3 \mathrm{~mm}$ it's highly signficat factor that makes difference in results between CTA and conventional coronary angiography assessment of the coronary stents.

In an overall view, regardless the site of examined stent, CT angiography compared to the conventional angiography as a gold standard technique in assessing patent coronary stent gave us a sensitivity of $92.3 \%$, a specificity of about $100 \%$, an accuracy of about $95.6 \%$, PPV is $100 \%$ and NPV is $90.5 \%$ taking into consideration that 2 stents were non evaluable due to narrow stent caliber $(2.5 \mathrm{~mm}$.) but proved patent by conventional angiography, Table (4).

Table (4): MDCT angiography statistical anaylsis of the results including the non available $(<3 \mathrm{~mm})$ stents

\begin{tabular}{|l|c|c|c|c|c|}
\hline \multirow{2}{*}{$\begin{array}{l}\text { Assessment } \\
\text { by CT }\end{array}$} & \multicolumn{5}{|c|}{ Assessment by angio } \\
\cline { 2 - 6 } & Accuracy & $\begin{array}{c}\text { Sensitivi } \\
\text { ty }\end{array}$ & $\begin{array}{c}\text { Specifici } \\
\text { ty }\end{array}$ & PPV & NPV \\
\hline Patent & $95.6 \%$ & $92.3 \%$ & $100.0 \%$ & $100.0 \%$ & $90.5 \%$ \\
\hline Occluded & $100.0 \%$ & $100.0 \%$ & $100.0 \%$ & $100.0 \%$ & $\begin{array}{c}100.0 \\
\%\end{array}$ \\
\hline$\geq 50 \%$ stenosis & $95.6 \%$ & $100.0 \%$ & $94.7 \%$ & $77.8 \%$ & $\begin{array}{c}100.0 \\
\%\end{array}$ \\
\hline$<50 \%$ stenosis & $95.6 \%$ & $50.0 \%$ & $100.0 \%$ & $100.0 \%$ & $95.3 \%$ \\
\hline Stenosis & $100.0 \%$ & $100.0 \%$ & $100.0 \%$ & $100.0 \%$ & $\begin{array}{c}100.0 \\
\%\end{array}$ \\
\hline
\end{tabular}

\section{Discussion}

Conventional coronary angiography has been considered the gold standard for evaluation of coronary artery stents and coronary artery bypass grafts. However, the main drawbacks of this method include invasiveness, patient discomfort, high radiation dose and risk of complications. A less invasive imaging modality is desirable for evaluation of patients suspected to have instent restenosis or occlusion and those who are suspected to have coronary artery bypass graft stenosis or occlusion.

Symptomatic patients who have undergone coronary stenting often pose a challenging diagnostic problem to the angiographer. The calcified, tortuous, and diffusely diseased coronary arteries complicate precise delineation of the lesions. High-quality angiographic images and thorough knowledge of the coronary anatomy are required in order to adequately determine revascularization options. Performing this angiographic evaluation in a noninvasive fashion is even more challenging. Noninvasive imaging techniques are hampered by specific limitations.

The major improvements of the recently developed 320- section CT machines compared with the old four-section scanners, include improved temporal resolution due to shorter gantry rotation time, better spatial resolution owing to sub-millimeter collimation, and considerably reduced scan acquisition times. Despite the recent technical advances, the 320detector row CT coronary angiography is still sensitive to arrhythmia. Persistent irregular cardiac rhythm such as atrial fibrillation and frequent extrasystoles preclude MDCT coronary angiography. However, motion artifacts owing to mild arrhythmia (e.g. single ventricular extra-systole) can be diminished by manual repositioning the reconstruction windows.

It is not advisable to scan patients whose BMI is above 40. In this study, the mean BMI for scanned patients was 29.76 with a range of 22-38. None of the performed scans was non-interpretable secondary to image noise as the BMI of the selected patients was still reasonable to perform coronary CTA.

Heart rate control is still essential even with the use of 320- row multislice CT scanner, not only to obtain good quality images, but also to lower radiation dose to the patients. The slower heart rate $(\leq 65 \mathrm{bpm})$ improves the temporal resolution and results in almost motion free images. It also permits the use of prospective ECG-gating.

In a pilot study done by Dewey et al. ${ }^{(2)}$ on 30 patients who underwent both coronary angiography 
and coronary CTA, they found that radiation exposure reduction was greatest in patients with heart rates $\leq 65 \mathrm{bpm}$, whereas the effective dose was significantly higher in those with higher heart rates because of the necessity of acquiring data over multiple cardiac cycles to increase temporal resolution, highlighting the importance of bblockade.

Andreini et al. ${ }^{(3)}$ study reported that slice misalignment due to inadequate heart rate control was another influential factor in ability to evaluate stents. MDCT feasibility was significantly lower in patients with heart rates $>60$ beats/min compared with $\leq 60$ beats/min.

In this study, heart rate control was achieved using oral medications: either Metoprolol or Ivabradine. With optimum heart rate control ( $\leq 65 \mathrm{bpm})$, we were able to scan the patients with prospective gating method exposing only $70-80 \%$ of $\mathrm{R}-\mathrm{R}$ interval using single beat acquisition at those with coronary stents. Only two cases failed to respond well to oral medications and their heart rate remained above 70 $\mathrm{bpm}$. So, we had to widen the scanning window to cover $30-80 \%$ of $\mathrm{R}-\mathrm{R}$ interval and to use two beats volume scanning for those with coronary stents. Even though, the obtained image quality was not satisfactory, while another one case had a premature beat at time of scan so we edit the ECG tracing and the reconstruction window during the premature heartbeat was deleted and another was added to the subsequent cardiac cycle. This step eliminate the abrupt heart rate change related to the premature beat and the in-stent lumen is well depicted on the multiplanar reformatted image showing a patent stent. Stent lumen visibility varies largely depending on stent type and diameter. The blooming effect is more disturbing in smaller coronary stents with thicker struts and is less disturbing in larger stents. Non-interpretable images tend to be obtained in stents that have a smaller diameter with thick sturts. When the stent diameter is more than $3 \mathrm{~mm}$, lumen visibility is better.

Regarding the type of stent, gold or gold-coated stents, along with tantalum made stents cause the most severe artifacts, while stainless steel and cobalt stents are better visualized ${ }^{(4)}$.

Carbone et al. ${ }^{(5)}$ evaluated the ability of 64- detector row CT to assess the coronary artery stent patency on fifty-five consecutive patients (age range 45-80 years) with 97 previously implanted coronary artery stents and the sensitivity, specificity, positive predictive value and negative predictive value were $75 \%, 86 \%, 71 \%$ and $89 \%$, respectively. However, nine of the 12 stented segments of $2.5 \mathrm{~mm}$ diameter and 10 of the 23 stented segments of $2.75 \mathrm{~mm}$ diameter were excluded from the analysis since these segments were considered as non-interpretable to blooming artifact.

A recently done study by Dawoud et al. ${ }^{(4)}$ using 320 MDCT on 24 patients with 63 stents, only 6 stents $(9.52 \%)$ were considered non-interpretable. Four of them are $2.5 \mathrm{~mm}$ in diameter while the remaining two are of $3.0 \mathrm{~mm}$ diameter. The difficulty in interpretation of $2.5 \mathrm{~mm}$ stents in this study was due to small stent caliber that makes accurate lumen visualization difficult. Their struts were thin and did not exert significant blooming artifact. One stent of the same diameter was not interpretable secondary to inadequate stent expansion which is probably a technical error during stent deployment. On the other hand, the two stents with $3.0 \mathrm{~mm}$ diameter were not interpretable secondary to thick struts that exert much blooming and beam hardening artifacts making lumen visualization difficult even with bone window settings, sharp reconstruction kernel and high $\mathrm{kV}$ and $\mathrm{mA}$ setting.

In this study among 50 patients with 65 stents only 2 stents (3\%) were non evaluable by MDCT. both of them are $2 \mathrm{~mm}$. the difficulty was also due to small stent caliber $\&$ thick stent struts that makes accurate lumen visualization difficult.

Another 2 stents in this study misinterpreted by MDCT. They are $3 \mathrm{~mm}$. in 2 different patients with heart rate $>65 \mathrm{bpm}$. due to anxious patient on the table despite of medical treatment. These stents diagnosed as ISR $\geq 50 \%$ where proved to be mild or $<50 \%$ stenosis by angiography.

The remaining 61 stents in this study were interpretable by MSCT and their lumen was clear 
enough to rule-out or diagnose in-stent restenosis. Some of those stents had thick struts that exert blooming artifact. However, the blooming artifact could be minimized by using sharp reconstruction kernel (dedicated edge-enhancing Kernel), bone window setting and small field of view (increasing the spatial resolution) and the lumen was clearly visualized.

A biodegradable stent with dense radio-opaque markers was evaluated by MSCT in this study, the blooming artifact exerted by those markers was not interfering with stent luminal assessment and instent restenosis was ruled out easily.

Based on stent location, stents located at proximal segments of RCA, LAD and LCx were better visualized than those deployed within the distal segments of the same arteries and also than those deployed within obtuse marginal and diagonal branches owing to large caliber of the former and small caliber of the later stents.

Cardiac motion artifact due to HR $>65 \mathrm{bpm}$ was a contributing factor in difficult stent lumen evaluation in this study at 2 stents $(3 \mathrm{~mm}$.) as the heart rate was $(70 \& 75 \mathrm{bpm}$.) due to anxious patient on the table. While the mean heart rate was $63 \mathrm{bpm}$ at the rest of patients which is considered slow enough to create motion free images and in the same studies that contain non-interpretable stent, the native coronary arteries were completely assessable. However, most authors stated that heart rate should be kept below $60 \mathrm{bpm}$ for better quality images when evaluating cases with coronary artery stents.

In this study, MSCT was capable not only of stent lumen evaluation to rule-out in-stent restenosis, but also was helpful in diagnosing significant disease at non-stented coronary arteries and detection of plaque composition either soft, calcified or mixed plaque. MSCT was capable of detecting serious incidental extra cardiac findings such as ascending aortic aneurysm that could be missed during conventional coronary angiography.

Although diagnostic accuracy of 320-row CTA may be comparable to the performance of 64-row scanners, advantages of this new technology lie in improved image acquisition as well as reduced radiation dose compared with retrospectively gated 64-row CTA. For the first time, since the introduction of CTA technology, $16 \mathrm{~cm}$ volumetric data acquisition within a single gantry rotation has become possible, allowing full cardiac imaging within a single gantry rotation, even in patients with an enlarged heart. Accordingly, single heart beat image acquisition allows for a significant reduction of contrast material and breath-hold time (with a total breath-hold time of $5 \mathrm{~s}$ ) when compared with CTA systems requiring multiple heart beats to image the entire heart ${ }^{(1)}$.

Furthermore, 320-row systems have increased temporal resolution ( $350 \mathrm{~ms}$ per gantry rotation) which reduces cardiac motion artefacts. Although certain types of 64-row systems have a slightly higher temporal resolution (330 ms per gantry rotation), these systems can only cover a small volume $(3.2 \mathrm{~cm})$ in a single heart beat. Similarly, dual-source systems, with even superior temporal resolution $(83 \mathrm{~ms})$, allow limited craniocaudal coverage per rotation. In contrast, 320-row CTA allows volumetric data acquisition with full cardiac coverage in a single rotation, eliminating the problem of stair-step artefacts associated with helical and step-and-shoot scanning techniques (1).

To reduce radiation dose in this study, we used prospective helical acquisition only exposing 70-80\% of $\mathrm{R}-\mathrm{R}$ interval in those with heart rate below 65 bpm, and $30-80 \%$ of $\mathrm{R}-\mathrm{R}$ interval in those with heart rate above $65 \mathrm{bpm}$.

Compared with conventional angiography, CT angiography is less costly, faster to perform, does not require assembly of an angiographic team to perform the study, generally available $24 \mathrm{~h}$ a day and can be considered as an outpatient procedure. It permits a wider variety of manipulations of the volumetric data set for image display and analysis in contrast to the limited projections routinely obtained during conventional angiography, and has fewer potential complications. Moreover, CT images can be reconstructed to yield $3 \mathrm{D}$ volume rendered images that give much anatomical and pathological details in contrast to conventional angiography that only visualizes lumen. 
Our study found that among the main causes of stent lumen un-evaluation by 320 -slice CT scanners, are the narrow stent calibers, thick stent struts, uncontrolled high heart rates, cardiac arrythmias and large body mass index.

By the aforementioned analysis of the calibers of the involved coronary stents within our study the results proves the direct relation between the caliber of the deployed stent and the right CT assessment of its patency with adequate $\mathrm{CT}$ visualization of the wide calibered stents, while increased suspicion of in-stent re-stenosis and failure of evaluation in small calibered stents $<3 \mathrm{~mm}$.

After exclusion of all non-evaluable stents, sensitivity, specificity, PPV, NPV, and accuracy for the detection of patent \& occluded stents and ISR regardless the percentage of stenosis were $100 \%$ due to the relatively small number of cases while in determining the percentage of in-stent restenosis in our study accuracy was $95.35 \%$.

In our study using 320-row CT scanner, the mean scan time was about $5 \mathrm{~s}$ (while it was $30 \mathrm{~s}$ with 16 section scanner and $20 \mathrm{~s}$ in 64 section scanner) thus improving image quality by acquiring data from fewer heart beats and the study becomes tolerable by most patients due to short breath hold time. The shorter scan time also allowed much reduction in the volume of contrast material usage.

Clinical evaluation of coronary arteries stents was performed by using multiplanar reformation of the data volume. Curved multiplanar reformation, cross-sectional images of the stent are the most useful views on which to assess patency, restenosis, or a minor degree of neointimal hyperplasia, maximum intensity projection, and with limited role of volume rendering images.

Wide window settings were necessary for accurate evaluation of the in-stent lumen at CT angiography (window width, $1500 \mathrm{HU}$; window center, $300 \mathrm{HU}$ ). The stent may be considered to be occluded if the lumen inside the device appears darker than the contrast-enhanced vessel lumen proximal to the stent not distal to it because collateral vessels may be feeding the vessel segment distal to the occluded stent in a retrograde direction.
Nonocclusive in-stent neointimal hyperplasia is characterized by the presence of a darker rim between the stent and the contrast-enhanced vessel lumen (Typically occurs as a localized nonenhancing lesion). If neointimal hyperplasia exceeds a luminal diameter reduction of $50 \%$ the process is consistent with hemodynamically significant in-stent restenosis.

\section{Conclusion}

Our study recommends usage of latest multidetector row CT scanners as a first-line tool for the noninvasive evaluation of patients with suspected instent restenosis specially with stents $\geq 3 \mathrm{~mm}$. and helps to identify factors that influence the assess ability of coronary artery stents by 320 MDCT scanners, namely, stent type and diameter.

\section{References}

1. de Graaf FR, Schuijf JD, van Velzen JE, et al. Diagnostic accuracy of 320-row multidetector computed tomography coronary angiography in the non-invasively assess in-stent restenosis. Investigative Radiology. 2010; 45(6): 1908-15.

2. Dewey M, Teige F, Schnapauff D, Laule $\mathrm{M}$, Borges AC, Wernecke KD, et al. Noninvasive detection of coronary artery stenoses with multislice computed tomography or magnetic resonance imaging. Ann Intern Med 2006; 145:407-15

3. Andreini D, Pontone G, Bartorelli AL, Trabattoni D, Mushtaq S, Bertella E, Annoni A, Formenti A, Cortinovis S, Montorsi P, Veglia F. Comparison of feasibility and diagnostic accuracy of 64slice multidetector computed tomographic coronary angiography versus invasive coronary angiography versus intravascular ultrasound for evaluation of in-stent restenosis. The American journal of cardiology. 2009; 103(10):1349-58. 
4. Dawoud MA, Alarabawy RA, Alla TM, Mubarak AA. Evaluation of coronary stents using multidetector CT. The Egyptian Journal of Radiology and Nuclear Medicine. 2016; 47(3):793-801.

5. Carbone I, Francone M, Algeri E, et al. Non-invasive evaluation of coronary artery stent patency with retrospectively ECGgated 64-slice CT angiography. Eur Radiol 2008; 18: 234-243. 